\title{
Toward the True Second Law \\ Part I: The Flaws in Clausius'Second Law Thermodynamics
}

\author{
José C. Íñiguez ${ }^{1}$ \\ ${ }^{1}$ Retired instructor of science and mathematics, $122721^{\text {st }}$ Street, Douglas AZ 85607, USA \\ Correspondence: José C. Íñiguez, Retired instructor of science and mathematics, $122721^{\text {st }}$ Street, Douglas AZ \\ 85607, USA. Tel: 1-520-368-2345. E-mail: iniguez.jose@gmail.com
}

Received: July 30, 2014 Accepted: August 7, 2015 Online Published: August 12, 2015

doi:10.5539/apr.v7n5p22 URL: http://dx.doi.org/10.5539/apr.v7n5p22

\begin{abstract}
Clausius' work leading to the law of increasing entropy -the supreme law of nature according to Eddington- is here shown to be a logically flawed construction. Both of the statements subsumed by this principle: the zero total entropy change for all reversible processes and the positive total entropy change for all irreversible processes are here disproved by counterexamples taken from the very premises of Clausius' work. In the second and last part of this work we will see how the second law of thermodynamics, as currently understood, emerges from the correction of the flaws in Clausius' work here made evident.
\end{abstract}

Keywords: Carnot's cycle, Carnot's theorem, Clausius' transformations, entropy, the second law of thermodynamics, counter-examples to the law of increasing entropy

\section{Carnot and the Motive Power of Fire}

\subsection{From Heat Engines to Lost Work}

The foundation of that parcel of knowledge which has the transformation of heat into work as its central theme was laid by Carnot in his 1824 memoir titled Reflections on the Motive Power of Fire, and on Machines Fitted to Develop that Power. Carnot's field of inquiry was to become -through the subsequent work of Clapeyron, Joule, Clausius, Thomson, and others- what is now known as second law thermodynamics'.

The opening paragraphs of Carnot's work - developed within the confines of the caloric theory of heat- describe the state of the art of the continuous generation of work (motive power) out of heat, as well as the challenges to be overcome to transform the said art into a science. In Carnot's own words:

"Every one knows that heat can produce motion. That it possesses vast motive-power no one can doubt, in these days when the steam-engine is everywhere so well known...Nature, in providing us with combustibles on all sides, has given us the power to produce, at all times and in all places, heat and the impelling power which is the result of it. To develop this power, to appropriate it to our uses, is the object of heat-engines... The study of these engines is of the greatest interest...they seem destined to produce a great revolution in the civilized world...(and even if) already the steam-engine works our mines, impels our ships, excavates our ports and our rivers, forges iron, fashions wood, grind grains, spins and weaves our clothes, (and) transports the heaviest burdens...their theory is very little understood, and the attempts to improve them are still directed almost by chance." (Carnot, 1824/1977, p. 3, 4, 5)

Carnot realized that in order to bring to light the principles governing the production of work in heat engines it was necessary to approach their study it in the most general way possible, i.e., "...independently of any mechanism or any particular agent..." this way it will be possible

“...to establish principles applicable not only to steam-engines but to all imaginable heat-engines, whatever the working substance and whatever the method by which it is operated." (Carnot, 1824/1977, p. 6)

Getting at the principles underlying work production in heat engines demanded of him the recognition of the essential elements required for this purpose:

"The production of motion in steam engines is always accompanied by a circumstance on which we should fix our attention. This circumstance is the... passage (of the caloric) from a body in which the temperature is more or less elevated, to another in which it is lower...We supply caloric to the first of these bodies that we may transmit it to 
the second by means of the intermediate substance... Heat can evidently be a cause of motion only on virtue of the changes of volume or form which it produces on bodies...Steam is a means to realizing this power, but it is not the only one. All substances in nature can be employed for this purpose, all are susceptible of changes of volume, of successive contradictions and dilatations, through the alternation of heat and cold. All are capable of overcoming in their changes of volume certain resistances, and of thus developing the impelling power... Reciprocally, wherever we can consume this power, it is possible to produce a difference of temperature..." (Carnot, 1824/1977, p. $6,8,9)$

This succession of "contradictions and dilatations" experienced by the work-producing, caloric-carrier intermediary substance was represented by Carnot in terms of the closed cycle of operations bearing his name. Carnot didn't provide a graphical representation of his cycle. It was in Clapeyron's 1834 paper Memoir on the Motive Power of Heat (Carnot, 1824/1977, p. 71) where the very first representation of Carnot's cycle, in $P-V$ coordinates (Carnot, 1824/1977, p. 75), was to appear.

Three things need here to be pointed out in regard to Carnot's previous quotes: (1) that the terms heat and caloric are used by him interchangeably (Carnot, 1824/1977, footnote p. 9); (2) that

"...the expression motive power... (designates) the useful effect that a motor is capable of producing. This effect can always be likened to the elevation of a weight to a certain height (and) has, as we know, as measure, the product of the weight multiplied by the height to which it is raised." (Carnot, 1824/1977, footnote p. 5); and (3) that the "... production of motive power is...due...not to an actual consumption of the caloric, but to its transportation from a warm body to a cold body..." (Carnot, 1824/1977, p. 7)

A hot and a cold bodies thermally connected by the cyclic path of changes of an intermediary substance came out in Carnot's work as the essential elements for work production in heat engines. The 'essential' qualifier can be understood by realizing that no heat engine can provide a continuous work output if any of these elements is missing.

The cycle by itself provided an explanation on the 'how' motion can be produced out of heat in heat engines (Carnot pp. 10-11; 17-19) Addressing the problem of 'how much' required, on its part, recognition of the factors having any saying on this matter. A comparison between work production in heat engines with that taking place by harnessing the potential energy of waterfalls led Carnot to the realization that paralleling the latter, the motive power of heat also depended on "... the quantity of caloric used..." and on what he called "...the height of its fall...", or in other words, on "the difference of temperature of the bodies between which the exchange of caloric is made." In regard to this latter factor, he elaborated as follows:

"In the waterfall the motive power is exactly proportional to the difference of level between the higher and lower reservoirs. In the fall of caloric the motive power undoubtedly increases with the difference of temperature between the warm and cold bodies; but we do not know whether it is proportional to this difference. We do not know, for example, whether the fall of caloric from 100 to 50 degrees furnishes more or less motive power than the fall of this same caloric from 50 to zero. It is a question which we propose to examine hereafter." (Carnot, 1824/1977, p. 15)

Before actually attempting to solve the question just raised, he proceeded to consider

"...this curious and important question: Is the motive power of heat invariable in quantity, or does it vary with the agent employed to realize it as the intermediary substance, selected as the subject of action of the heat?", adding "It is clear that this question can be asked only in regard to a given quantity of caloric, the difference of the temperatures also being given." (Carnot, 1824/1977, p. 9)

From what has been stated above it should here be understood that the difference of temperatures he is referring to, involves two specific temperatures, i.e., a temperature difference at a specific location in the temperature scale. Providing an answer to this "curious" question started with Carnot's identifying the direct transfer of caloric from the hot to the cold body as a factor decreasing the work output of heat engines: "Every change of temperature which is not due to a change of volume... is necessarily due to the direct passage of the caloric from a more or less heated body to colder body. This passage occurs mainly by the contact of bodies of different temperatures; hence such contact should be avoided as much as possible..." because it brings "...loss of motive power." In reference to this contact he further stated: "It cannot probably be avoided entirely, but it should at least be so managed that the bodies brought in contact with each other differ as little as possible in temperature." (Carnot, 1824/1977, p. $13,14)$ The previous considerations were summarized in what Carnot calls the necessary condition for the attainment of the maximum possible output of work: "...that in the bodies employed to realize the motive power of heat there should not occur any change of temperature which may not be due to a change of volume...every 
time this condition is fulfilled the maximum will be attained." (Carnot, 1824/1977, p. 13) It was to the determination of this maximum of work that under a given set of conditions of heat flow and temperature fall an engine can output, where Carnot's efforts were directed to.

\subsection{Heat Engines, Refrigerators, and Carnot's Theorem}

As already noted, Carnot knew that the consumption of work in an inverse cycle is accompanied by the transfer of caloric from the cold to the hot body. Any inverse cycle can at this light reverse in larger or lesser degree the effects of the heat engine preceding it. This idea follows naturally from the fact that heat-work inter-conversions in heat engines come on reason of changes of volume. If the direction of the volume change is reversed so will be the direction of the other changes involved. For example in an expansion the variable body receives heat from a source and transforms it into work. If this process is reversed, work will be consumed and heat returned back to the source. The extrapolation of this notion to a cyclical process producing the maximum of motive power convinced him that here, in the absence of lost work, a perfect reversal or cancelation of effects had to take place. In reference to a heat engine producing work out of the transfer of caloric from a hot body $A$ to a cold body $B$, he states:

"The result of these first operations has been the production of a certain quantity of motive power and the removal of caloric from the body $A$ to the body B. The result of the inverse operations is the consumption of the motive power produced and the return of the caloric from the body B to the body A; so that these two series of operations annul each other, after a fashion, one neutralizing the other." (Carnot, 1824/1977, p. 19)

The previous statement, it should be noted, constitutes what is perhaps the most general definition of reversibility. It should be compared with that advanced later on by Planck defining reversibility in terms of the possibility "...to restore everywhere the exact initial state once the process has taken place.” (Planck 1990, p. 84) The veracity of Carnot's previous statement -the perfect cancelation of effects- came by way of showing, via a reductio ad absurdum argument, that any assumption on the contrary leads to a known false conclusion: the possibility of perpetual motion, understood here as the creation of motive power out of nothing (Carnot (Clapeyron), p. 74) In his own words:

"...if there existed any means of using heat preferable to those which we have employed, that is, if it were possible by any method whatever to make the caloric produce a quantity of motive power greater than we have made it produce by our first series of operations, it would suffice to divert a portion of this power in order by the method just indicated to make the caloric of the body B return to the body A from the refrigerator (cold body) to the furnace, to restore the initial conditions, and thus be ready to commence again an operation precisely similar to the former, and so on: this would be not only perpetual motion, but an unlimited creation of motive power without consumption either of caloric or of any other agent whatever. Such a creation is entirely contrary to the ideas now accepted, to the laws of mechanics, and of sound physics. It is inadmissible." (Carnot, 1824/1977, pp. 11-12)

This result allowed him to voice his answer to the said "curious question" in the following form:

"The motive power of heat is independent of the agents employed to realize it; its quantity is fixed solely by the temperatures of the bodies between which is effected, finally, the transfer of the caloric." (Carnot, 1824/1977, p. 20)

The previous statement is known as Carnot's theorem. It represents the first of the principles underlying work production in heat engines that he set out to identify. From it we learn that for a given temperature difference and amount of caloric, no particularity introduced in a reversible heat engine, be it in the form of a different intermediary substance, in the form of a different cyclical path for the working substance, or any other conceivable modification, can make it produce an amount of work larger than the maximum. This result is usually stated by saying that no engine can be more efficient than a reversible Carnot engine. Carnot's theorem might be considered as the first statement of the second law of thermodynamics.

At this point in his analysis only one piece of the puzzle remained: that of establishing a functional relation between maximum work output and the temperatures of the reservoirs. The solution of this problem was to produce the second -and last- of the principles governing work production in heat engines. Its solution depended on finding a functional relation between work output, amount of caloric flowing, and the temperature of the hot and cold bodies. He searched for it using an 'infinitesimal cycle' as analytical device (Carnot, 1824/1977, pp. 20-21). This, his only attempt -consigned in his memoir to a footnote (Carnot, 1824/1977, pp. 37-40)- failed to produce the expected results (Müller, 2007, p. 54).

Even if Carnot was capable of recognizing the essence of the connection between work and temperature, its mathematical form eluded him. Referring to the position of a given temperature difference in the thermometric 
scale he understood that "... a given quantity of heat will develop more motive power in passing from a body kept at 1 degree to another maintained at zero, than if these two bodies were at the temperature of $101^{\circ}$ and $100^{\circ}$ ", in other words, that "...the fall of caloric produces more motive power at inferior than at superior temperatures..." (Carnot, 1824/1977, p. 36, 37) Beyond this he recognized that even if "We have shown that the quantity of motive power developed by the transfer of caloric from one body to another depends essentially on the temperatures of the two bodies... we have not shown the relation between these temperatures and the quantities of motive power produced." (Carnot, 1824/1977, p. 35), concluding with "We are not prepared to determine precisely, with no more experimental data that we now possess, the law according to which the motive power of heat varies at different points on the thermometric scale." (Carnot, 1824/1977, p. 37).

The solution to this problem: the mathematical definition of the reversible efficiency, among others, was accomplished by Clausius about a quarter of a century after the publication of Carnot's memoir.

In addition to Müller, further comments on Carnot's work and related issues can be found in Callendar (1911, pp. 153-189), Jaynes (1988, pp. 267-281), Purrington (1997, pp. 75-101), and Truesdell (1980, pp. 79-137)

\section{Clausius and the Mechanical Theory of Heat}

\subsection{Clausius Modifies some of Carnot's Assumptions}

Clausius introduced two critical modifications into Carnot's work. He abandoned the caloric theory of heat underwriting it, and chose a different basis of proof for his results. The first of these modifications brought Carnot's analysis in compliance with the results of Joule and others about the equivalence of heat and work, knowledge at the center of what was to be known as the first law of thermodynamics. From this perspective, Carnot's assertion that in the process of work production in heat engines heat was transferred but not consumed had to be abandoned. Clausius explained it as follows:

"...This mode of dealing with the question does not accord with our present views, inasmuch as we rather assume that in the production of work a corresponding quantity of heat is consumed, and that in consequence the amount of heat given out to the surrounding space during the cyclical process is less than that received by it. Now if for the production of work heat is consumed, then...there is no ground whatever for saying that the work is created out of nothing. Accordingly not only must the principle enunciated by Carnot receive some modification, but a different basis of proof from that used by him must be discovered." (Clausius, 1879, p. 77)

This new basis of proof he found in the principle:

"Heat cannot, of itself, pass from a colder to a hotter body" (Clausius, 1879, p. 78).

Armed with these two notions he was able first to reword, and then to prove the correctness of Carnot's theorem in its new form. The proof carried on by Clausius, identical in essence to the reductio ad absurdum argument previously used by Carnot, 1824/1977, showed that denying the validity of Carnot's corrected theorem implied the unassisted transference of heat from a colder to a hotter body, result that stands in direct contradiction with experience (Clausius, 1879, pp. 79-81). Clausius rewording of Carnot's theorem took the following form

“...the relation between the quantity of heat carried over, and that converted into work, is independent of the nature of the matter which forms the medium of the change... it can only depend on the temperature of the two bodies... which act as heat reservoirs." (Clausius, 1879, p. 79, 81)

In accord with this new knowledge, Clausius described one cycle in the operation of a reversible cycle (either an engine or a refrigerator) as follows:

"...two variations in respect to heat take place, viz. that a certain quantity of heat is converted into work (or generated out of work), and another quantity of heat passes from a hotter into a colder body (or vice versa)." Adding that "For the former of these two variations we have already employed the word 'transformation,' inasmuch as we said, when work was expended and heat thereby produced, or conversely when heat was expended and work thereby produced, that the one had been 'transformed' into the other. We may use the word 'transformation' to express the second variation also (which consists in the passage of heat from one body into another, which may be colder or hotter than the first), inasmuch as we may say that heat of one temperature 'transforms' itself into heat of another temperature."

With these antecedents in place, he proceeded first to “...describe the result of a simple cyclical process in the following terms: Two transformations are produced, a transformation from heat into work (or vice versa) and a transformation from heat of a higher temperature to heat of a lower (or vice versa)..." and then to recognize "The relation between these two transformations...(as) that which is to be expressed by the second Main Principle" (Clausius, 1879, pp. 91-92). 
The representation of one cycle in the operation of a reversible engine according to Clausius previous description is given in Figure 1.

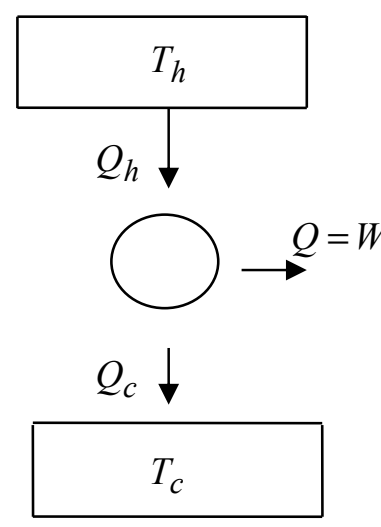

(a)

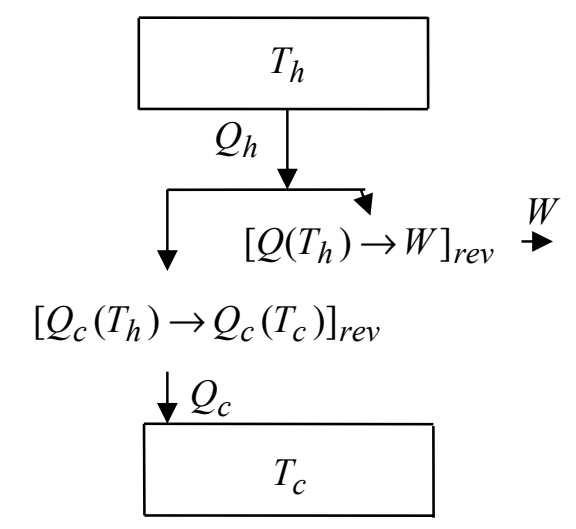

(b)

Figure 1. Two equivalent renditions of a Carnot reversible engine are here shown according to the modifications introduced by Clausius. Both of them highlight the streams of heat transferred from the hot to the cold reservoir, as well as that which originating in the hot reservoir, ends up as work in the mechanical reservoir. Here $Q_{h}-Q_{c}=Q=W$. In figure $(b)$ use has been made of the transformation notation introduced in section 2.4

Notwithstanding the central role played by these transformations in Clausius' analysis, he never actually identified the process or processes responsible for each of them. In being essential in terms of the forthcoming critiques to Clausius' results on this matter, this identification will be performed in section 2.4.

All but one of Clausius quotes come from his book The Mechanical Theory of Heat. The reader will find that the transcription of most of these quotes include slight changes in notation, introduced to render them more familiar.

\subsection{Carnot's Reversible Cycle}

The essential elements of Carnot's reversible cycle have been depicted in Figure 2. There we find: (1) A hot reservoir of temperature $T_{h}$; (2) A cold reservoir of temperature $T_{c}$, with $\left.T_{h}\right\rangle T_{c}$; (3) The cyclical path of the working substance or variable body, here assumed to be an ideal gas, and finally, (4) A mechanical reservoir (MR) which can be assumed to be of the weight-in-pulley kind.

The description of the cycle's constitutive processes given below is made in reference to Figure 2(a) and follows current usage.

1) Process $A B$ represents an isothermal expansion at the temperature $\left(T_{h}\right)$ of the hot reservoir. Here an amount of heat $Q_{h}$ transferred from the hot reservoir to the gas ends up being transformed, via the reversible expansion, into an equivalent amount of work $W_{h}$. This work appears in the form of a potential energy increase of the weight in the mechanical reservoir.

2) Process $B C$ represents an adiabatic expansion. Here the gas performs work at the expense of its internal energy and its temperature falls from $T_{h}$ to $T_{c}$

3) Process $C D$ is an isothermal compression at the temperature $\left(T_{c}\right)$ of the cold reservoir. Here the amount of work $W_{c}$ supplied by the mechanical reservoir to carry on this process ends up as the equivalent amount of heat $Q_{c}$ in the cold reservoir.

4) Process $D A$, the one that closes the cycle by bringing the variable body to its initial condition $A$, is an adiabatic compression. The work expended in carrying on this process ends up increasing the internal energy of the gas and thus raising its temperature from $T_{c}$ to $T_{h}$. 


\subsection{An Interlude}

Most of the thermodynamic notions appearing in Clausius' quotes are either explicit or implied in Chapter 2 of The Mechanical Theory of Heat (Clausius, 1879, pp. 39-68). To avoid repetition some of these will be here either pointed out, justified, or proved.

1) As Carnot originally realized (p. 18), in the ideal-gas isothermal and reversible volume changes taking place in the cycle, the work involved -either produced in an expansion or consumed in a compression- is larger the larger the temperature. In reference to Figure 2 this translates as $\left.W_{h}\right\rangle W_{c}$. The difference between the work respectively produced and consumed along the hot and cold isotherms constitutes the work output of the cycle, $W=W_{h}-W_{c}$.

2) The internal energy $(U)$ of ideal gases is a sole function of the absolute temperature.

3) From the previous notion it follows that for any isothermal change of an ideal gas the first law of thermodynamics reduces to $Q=W$

4) From notions (1) and (3) it follows, using the notation of Figure 2, that $Q_{h}>Q_{c}$. The difference between the amounts of heats respectively released and absorbed by the hot and cold reservoirs corresponds with the net work output of the cycle, $Q_{h}-Q_{c}=Q=W$

5) On reason of the $Q=0$ constraint that define their evolution, the first law for adiabatic and reversible processes $B C$ and $D A$ of Figure 2, reduces to $\Delta U=-W$. For these processes it is true that $\Delta U_{B C}=C_{v}\left(T_{c}-T_{h}\right), \Delta U_{D A}=-C_{v}\left(T_{c}-T_{h}\right)$, and also true that $W_{B C}=-C_{v}\left(T_{c}-T_{h}\right), W_{D A}=C_{v}\left(T_{c}-T_{h}\right) . \mathrm{A}$ simple inspection of the previous results makes evident that any one of these processes is the inverse of the other in the sense that the effects of one are precisely canceled by the other, fact that comes to light in the following equations: $\Delta U_{B C}+\Delta U_{D A}=0, W_{B C}+W_{D A}=0$.

\subsection{Carnot's Reversible Cycle and Clausius Transformations}

Let us now center our attention on Figure 2(a) and based on the fact that the heat $\left(Q_{h}\right)$ taken in by the ideal gas along $A B$ is larger than that $\left(Q_{c}\right)$ it gives out to the cold reservoir along $C D$ conclude that, as indicated in Figure 2(b), there exists some point $E$ on isotherm $A B$ such that in its transit $E B$ the ideal gas takes from the hot reservoir an amount of heat $Q_{c}$ identical in magnitude to the one it transfers to the cold reservoir along $C D$. The approximate location of this point is shown in Figure 2(b).

Assuming that the operation is to start at point $E$, consideration will be given to the concatenation of processes $E B-B C-C D-D A$. Actually, and on reason of the previously noted inverse relation existing between processes $B C$ and $D A$, we will circumscribe our attention to processes $E B$ and $C D$.

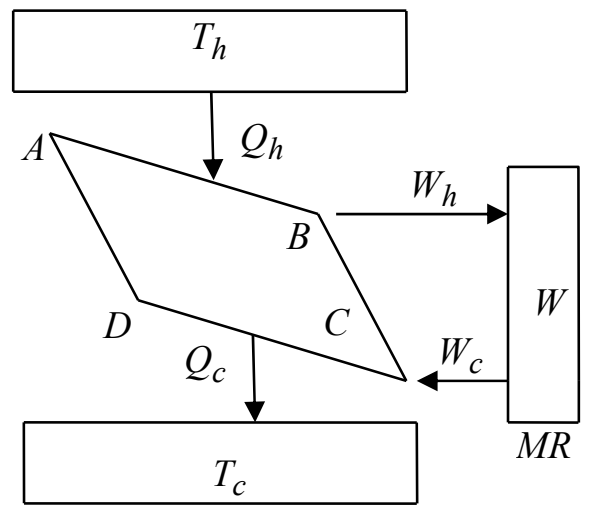

(a)

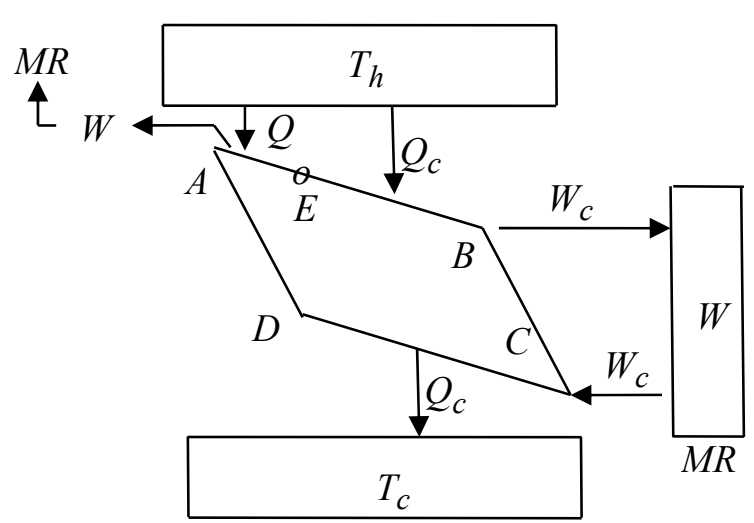

(b)

Figure 2. These two graphical descriptions of Carnot's reversible cycle of operations accord to the modifications introduced by Clausius. They differ only in the starting point of the cycle. While in (a) the cyclical path of the variable body is $A-B-C-D-A$, in (b) is $E-B-C-D-A-E$. The latter, described in detail in Section 2.4, will be used in the identification of the values or entropy changes of the two transformations with which Clausius represents the effects of the cycle. In these figures $W_{h}-W_{c}=W, Q_{h}-Q_{c}=Q, Q=W$ 
Along isothermal and reversible expansion $E B$ an amount of heat $Q_{c}$ of temperature $T_{h}$ transferred by the hot reservoir to the ideal gas is transformed into an equivalent amount of work $W_{c}$. This work appears in the form of a potential energy increase of the weight in the mechanical reservoir. Along isothermal and reversible compression $C D$, on the other hand, the amount of work $W_{c}$ supplied by the mechanical reservoir to carry on this process ends up in the cold reservoir as an equivalent amount of heat $Q_{c}$ of temperature $T_{c}$. The fact that at the end of this series of changes the mechanical reservoir, in releasing the amount of work it had previously received, recuperates its initial condition, leaves the transfer of $Q_{c}$ from the hot to the cold reservoir as the sole effect of concatenation $E B-B C-C D-D A$. For reasons of economy and clarity of expression the effect produced by this sequence of processes will be represented via the following self-evident notation: $\left[Q_{c}\left(T_{h}\right) \rightarrow Q_{c}\left(T_{c}\right)\right]_{r e v}$.

The reversible quality of this transformation, highlighted by the ' $r e v$ ' sub index, comes to light if we subject the variable body to the sequence of processes $A D-D C-C B-B E$. Here process $D C$ represents an expansion. Through it an amount of heat $Q_{c}$ absorbed from the cold reservoir ends up as an equivalent amount of work $W_{c}$ in the mechanical reservoir. Process $B E$ is, on its part, a compression. The work $W_{c}$ expended in carrying it to completion ends up in the hot reservoir as an equivalent amount of heat $Q_{c}$. At the end the mechanical reservoir recuperates its initial condition, leaving the transfer of $Q_{c}$ from the cold to the hot reservoir as the sole effect of this concatenation of processes. This transformation, one of the two associated to the operation of a reversible refrigerator, will be from now on represented as $\left[Q_{c}\left(T_{c}\right) \rightarrow Q_{c}\left(T_{h}\right)\right]_{\text {rev }}$. Subsumed in the reversal of effects just shown is the fact that running one of these series of operations after the other will restore the initial condition. For example, if this last series of operations is run once the former has concluded, we will find the cold reservoir losing the amount of heat it has previously received; the hot reservoir recuperating the one it initially lost; and the mechanical reservoir in its original condition. At the end of this coupling of processes no change remains. This is what makes both of these transformations reversible.

Going back to figure 2(b) we have that once the concatenation of processes $E B-B C-C D-D A$ has come to an end, the variable body finds itself at point $A$. All that is needed to bring the cycle to completion is the performance of isothermal and reversible expansion $A E$. Through it an amount of heat $Q$ of temperature $T_{h}$ transferred by the hot reservoir to the ideal gas ends up as an equivalent amount of work $W$ in the mechanical reservoir. The fact that no other effect but this one is here produced, allows us to identify isothermal and reversible expansion $A E$ as the one responsible for the heat-to-work effect of the reversible cycle, same that will be from now on represented as $\left[Q\left(T_{h}\right) \rightarrow W\right]_{r e v}$. An argument similar to that just presented in regard to the reversibility of $\left[Q_{c}\left(T_{h}\right) \rightarrow Q_{c}\left(T_{c}\right)\right]_{r e v}$ can be advanced to illustrate the fact that following process $A E$ with process $E A$ implies retrieving the work originally deposited in the mechanical reservoir and with it compressing the variable body; the spent work appearing as the equivalent amount of heat $Q$ in the hot reservoir. At the end of this coupling of processes no change remains. The original condition has been restored and these processes are reversible. This argument allows us to realize that the sole effect of process $E A$ consists in the reversible transformation of $W$ into the equivalent amount of heat $Q$, or equivalently in $\left[W \rightarrow Q\left(T_{h}\right)\right]_{\text {rev }}$

\subsection{Clausius Solution to the Efficiency Problem}

To address the problem whose solution evaded Carnot, 1824/1977, Clausius made use of his just proven version of Carnot's theorem: “...the relation between the quantity of heat carried over, and that converted into work, is independent of the nature of the matter which forms the medium of the change... it can only depend on the temperature of the two bodies... which act as heat reservoirs." (Clausius, 1879, p. 79, 81)

Being work production the primary goal of a heat engine it is only logical to expect that its efficiency be measured by the ratio between the heat transformed into work $(Q)$ and the heat released by the hot reservoir $\left(Q_{h}\right)$, i.e. $Q / Q_{h}$. Alternative measures of efficiency can, however, be defined according to which heat stream the emphasis is on. Among these we find the one mentioned in the corrected theorem, $Q_{c} / Q$, this is, the ratio between the heat carried over and that converted into work, and also $Q_{h} / Q_{c}$, the ratio between the amounts of heat respectively released and received by the hot and cold reservoirs. Any of these efficiency measures can be obtained from any other by recalling that these amounts of heat are related by the following equation

$$
Q_{h}-Q_{c}=Q
$$

It was the $Q_{h} / Q_{c}$ ratio the one actually used by Clausius to give mathematical expression to the corrected theorem. The resulting expression, $Q_{h} / Q_{c}=\phi\left(T_{h}, T_{c}\right)$, a statement of the sole dependence of this ratio on the temperatures of the heat reservoirs, was the starting point of Clausius efficiency analysis. The function $\phi$ linking ratio and temperatures was described by him as "...some function of the two temperatures which is independent of the nature of the variable body." (Clausius, 1879, p. 81). It was the unveiling of $\phi$ what was to lead Clausius to the solution of the efficiency problem, or equivalently, to the identification of the mathematical relation between 
maximum work output and the temperatures of the reservoirs. Clausius next step consisted in the selection of a suitable variable body with which to carry on the analysis leading to the identification of $\phi$ :

"The circumstance that (this) function...is independent of the nature of the variable body, offers a ready means of determining this function, since as soon as we have found its form for any single body it is known for all bodies whatsoever...Of all classes of bodies the perfect gases are best adapted for such a determination, since their laws are the most accurately known. We will therefore consider the case of a perfect gas subjected to a cyclical process..." (Clausius, 1879, p. 81)

With this notion at hand, and based on his knowledge of the laws governing the behavior of ideal gases (Clausius, 1879, pp. 60-68), he established the appropriate relations between the initial and final volumes of adiabatic and reversible processes $B C$ and $D A,\left(T_{h} / T_{c}\right)=\left(V_{C} / V_{B}\right)^{\gamma-1},\left(T_{h} / T_{c}\right)=\left(V_{D} / V_{A}\right)^{\gamma-1}$, to obtain, via a simple ratio, the following equation (Clausius, 1879, p. 82)

$$
\frac{V_{B}}{V_{A}}=\frac{V_{C}}{V_{D}}
$$

He also knew that the evolution of isothermal and reversible processes $A B$ and $C D$ were governed by the following equations (Clausius, 1879, pp. 82-83)

$$
\begin{aligned}
& Q_{h}=R T_{h} \ln \frac{V_{B}}{V_{A}} \\
& Q_{c}=R T_{c} \ln \frac{V_{c}}{V_{D}}
\end{aligned}
$$

Via the simple procedure of substituting Equation (2) in (3), followed by division of Equation (3) by Equation (4), he was able to write the explicit version of $Q_{h} / Q_{c}=\phi\left(T_{h}, T_{c}\right)$ as

$$
\frac{Q_{h}}{Q_{c}}=\frac{T_{h}}{T_{c}}
$$

From which he was able to identify the function $\phi$ as

$$
\phi=\frac{T_{h}}{T_{c}}
$$

At the light of Clausius procedure leading to Equation (5) it is only logical to assume that the reason for him choosing $Q_{h} / Q_{c}$ over $Q / Q_{h}$ or $Q_{c} / Q$ to define his function of interest, $Q_{h} / Q_{c}=\phi\left(T_{h}, T_{c}\right)$, was the fact that the former ratio is the one arising naturally from the equations used. Equivalent re-expressions of Equation (5) in terms of the two other efficiency quotients can be obtained via its appropriate combination with Equation (1). By doing this we get

$$
\begin{aligned}
\frac{Q_{c}}{Q} & =\frac{T_{c}}{T_{h}-T_{c}} \\
\frac{Q}{Q_{h}} & =\frac{T_{h}-T_{c}}{T_{h}}
\end{aligned}
$$

The previously noted fact that $Q=W$, allows us writing the previous equation as follows

$$
W=Q_{h} \frac{T_{h}-T_{c}}{T_{h}}
$$

Even if Equations (5), (7), (8), and (9), are indeed efficiency equations, it is this last one, Equation (9), the one which closely reflects Carnot's foresight regarding the factors determining the motive power of heat in heat engines. Keeping in mind the caloric theory of heat permeating his ideas, we can recognize in $Q_{h}$ the "...amount of caloric used...", and in $\left(T_{h}-T_{c}\right) / T_{h}$ not only the "...height of its fall..." but also the effect that the position of this temperature fall in the thermometric scale has on work production (Carnot, 1824/1977, p. 15). Note in this regard that for given values of $Q_{h}$ and $\left(T_{h}-T_{c}\right)$, larger amounts of work will be outputted for progressively smaller values of $T_{h}$, or in Carnot's words: "The fall of caloric produces more motive power at inferior than at superior temperatures." (Carnot, 1824/1977, p. 36) As written, Equation (9) embodies the second and last of the principles governing work production of heat engines i.e., the same that evaded Carnot's efforts. Combined Carnot's theorem 
(Section 1.2) and Clausius' Equation (9) subsume the essential knowledge about work production in heat engines: Its independence of the nature of the variable body, as well as its relation with the temperatures of the reservoirs.

Equation (9) is commonly known as the efficiency $\left(\eta_{r e v}\right)$ equation for a reversible heat engine working between two heat reservoirs of temperatures $T_{h}$ and $\left.T_{c}, T_{h}\right\rangle T_{c}$

$$
\eta_{\text {rev }}=\left(Q / Q_{h}\right)_{\text {rev }}=\left(W / Q_{h}\right)_{r e v}=\frac{T_{h}-T_{c}}{T_{h}}
$$

The connection between Equations (5) and (7) with Equation (10) can be unveiled via simple algebraic manipulations. By doing this we get $Q_{c} / Q_{h}=1-\eta_{r e v}$ and $Q / Q_{c}=W / Q_{c}=\eta_{\text {rev }} \frac{T_{h}}{T_{c}}$. That the solution to the 'how much' work a reversible cycle came in the form of Equation (5), an efficiency related equation, justifies the name of this section.

The long journey initiated by Carnot reaches, through the efforts of Clausius, its fruitful conclusion with Equation (5) and its re-expression, Equation (9). The theoretical frame of the science of heat engines, as Carnot had envisaged it, was now complete. As this was happening, a new field of inquiry, with Equation (5) at its center, was being opened by Clausius. His work in this direction led to the introduction of the entropy function, to the recognition of the law of increasing entropy, and to the prediction of the ultimate fate of the universe in the form of its heat death. No small feat for a body of knowledge having a furnace, a condenser, and steam as protagonists.

\subsection{The Entropy Changes for Clausius Transformations}

With the efficiency problem out of the way, the only issue remaining for Clausius to bring his study of heat engines to a satisfactory conclusion was the one related to the quantification of the effects of his transformations. In order to understand Clausius solution to this problem we will start by writing Equation (5) in the following manner

$$
-\frac{Q_{h}}{T_{h}}+\frac{Q_{c}}{T_{c}}=0
$$

Upon its combination with Equation (1) it takes the following form

$$
\left[-\frac{Q_{c}}{T_{h}}+\frac{Q_{c}}{T_{c}}\right]-\left[\frac{Q}{T_{h}}\right]=0
$$

As noted in Section 2.1, Clausius recognized two as the effects remaining once a cycle in the operation of a reversible engine comes to its conclusion: the transference to the cold reservoir of the portion $Q_{c}$ out of the amount of heat $Q_{h}$ released by the hot reservoir, and the transformation into work of the remaining portion, $Q=W=Q_{h}-Q_{c}$.

A simple inspection of the Equation (12) brings to light the fact that while the left parenthesis makes exclusive reference to the amount of heat carried over $\left(Q_{c}\right)$ as well as to the temperatures of the reservoirs involved in the transfer; the other one makes exclusive reference to the amount of heat $(Q)$ transformed into work as well as to the temperature of the reservoir in which this heat originates.

Judging from the way Clausius reasoned his way through to what he called 'the equivalence values' of the transformations -made evident in the quotes below- with 'value' meaning the quantifier of the effect of a transformation, a term that was to be eventually replaced by 'entropy change', it is only reasonable to assume that the pairing of Equation (12) with the effects of the transformations suggested Clausius that those parenthetical expressions were the actual values of said transformations.

The train of thought that actually took Clausius to the values of his transformations started with him recognizing that "...the value of a change from work into heat must be proportional to the quantity of heat generated, and ...beyond this it can only depend on its temperature." In regard to the transformation of heat from one temperature to another his reasoning went as follows: "Similarly the value of the passage of a quantity of heat $Q_{c}$ from the temperature $T_{h}$ to the temperature $T_{c}$ must be proportional to the quantity of heat which passes, and beyond this can only depend on the two temperatures" (Clausius, 1879, p. 98).

On this matter, he further elaborated as follows:

"...the passage of the quantity of heat $Q_{c}$ from temperature $T_{h}$ to $T_{c}$ has the same equivalence-value as a double transformation of the first kind, viz. the transformation of the quantity $Q_{c}$ from heat of temperature $T_{h}$ into work, and again out of work into heat of temperature $T_{c}$.", concluding with: "...in any case we may, in the 
mathematical determination of the Equivalence-Value, treat every transference of heat, in whatever way it may have taken place, as a combination of two opposite transformations of the first kind..." (Clausius, 1879, p. 101)

Translation of the previous considerations into mathematical expressions followed by further mathematical manipulation allowed Clausius to produce the values for his reversible transformations (Clausius, 1879, pp. 98101). He expressed his results in the following manner:

"Hence the second Main principle of the Mechanical Theory of Heat, which in this form may perhaps be called the principle of the Equivalence of Transformations, can be expressed in the following terms: If we call two transformations which may cancel each other without requiring any other permanent change to take place, Equivalent Transformations, then the generation out of work of the quantity of heat $Q$ of temperature $\tau$ has the equivalence-value $Q / \tau$; and the transference of the quantity of heat $Q$ from temperature $T_{1}$ to temperature $T_{2}$ has the Equivalence-value $Q\left(\frac{1}{\tau_{2}}-\frac{1}{\tau_{1}}\right)$; in which $\tau$ is a function of temperature independent of the kind of process by which the transformation is accomplished." (Clausius, 1879, pp. 100-101)

His eventual identification of $\tau$ with the absolute temperature $T$ (Clausius, 1879, pp. 107-108) makes the previous values identical with those hinted by Equation (12).

Equation (12) not only displays the values for the two transformations, it also shows that on reason of their values having the same magnitude but different sign, they add up to zero.

\subsection{The Entropy Function}

Clausius saw in the cancellation of values made evident by Equation (12) a property common to reversible cyclical processes. He extended his analysis to reversible cyclical processes of increased complexity. For those with an arbitrary number of constant temperature heat reservoirs he found, in correspondence with Equation (12), that the combined value of all the transformations was equal to $\sum(Q / T)=0$; while for those in which "...the temperatures of the heat reservoirs vary continuously...(so that) the quantities of heat given off and taken in (are) distributed in indefinitely small elements..." he found that the summation of values was in turn given by the expression $\int \frac{d Q}{T}=0$ (Clausius, 1879, pp. 101-109), about which he had the following to say:

"This equation, which was first published by the author in 1854 (Pogg. Ann. Vol. 93, p. 500) forms a very convenient expression for the second main Principle of the Mechanical Theory of Heat, as far as it relates to reversible processes. This principle may be expressed in words as follows: If in a reversible Cyclical Process every element of heat taken in (positive or negative) be divided by the absolute temperature at which is taken in, and the differential so formed be integrated for the whole course of the process, the integral so obtained is equal to zero." (Clausius, 1879, p. 90)

\section{Recognizing as well that}

"If the integral $\int d Q / T$, corresponding to any given succession of variations of a body, be always equal to zero provided the body returns finally to its original condition, whatever the intervening conditions may be, then it follows that the expression under the integral sign, viz. $d Q / T$, must be the perfect differential of a quantity, which depends only on the present condition of a body, and is altogether independent of the way in which it has been brought into that condition. If we denote this quantity by $S$, we may put $d Q / T=d S$, or $d Q=T d S$, an equation which forms another expression, very convenient in the case of certain investigations, for the second main principle of the Mechanical Theory of Heat" (Clausius, 1879, p. 90)

This quotient of reversible heat divided by the absolute temperature at which it is exchanged received from Clausius the name entropy, from the Greek word: $\pi \rho \circ \pi \eta$ : transformation. (Clausius, 1879, p. 107) For any given body reversibly exchanging heat at constant temperature, v. gr. a heat reservoir, the entropy change is given by the integrated form of $d S=d Q / T$, namely

$$
\Delta S=Q_{\text {rev }} / T
$$

In line with Clausius convention (Clausius, 1879, p. 97), the change will be positive if heat is taken in, and negative if given out.

A comparison between the values for the transformations given in Clausius last quote of section 2.6 and the definition just given for entropy change allows us to realize that the two terms are equivalent. According to this, the entropy changes of the two transformations produced by one cycle in the evolution of a reversible heat engine can be written, using the self-evident 'transformation notation' introduced in Section 2.4, as follows: 


$$
\begin{gathered}
\Delta S_{\text {Clausius }}\left[Q\left(T_{h}\right) \rightarrow W\right]_{\text {rev }}=-\frac{Q}{T_{h}} \\
\Delta S_{\text {Clausius }}\left[Q_{c}\left(T_{h}\right) \rightarrow Q_{c}\left(T_{c}\right)\right]_{\text {rev }}=-\frac{Q_{c}}{T_{h}}+\frac{Q_{c}}{T_{c}}
\end{gathered}
$$

While the two associated to its reversible inverse (refrigerator) take the following form

$$
\begin{gathered}
\Delta S_{\text {Clausius }}\left[W \rightarrow Q\left(T_{h}\right)\right]_{\text {rev }}=\frac{Q}{T_{h}} \\
\Delta S_{\text {Clausius }}\left[Q_{c}\left(T_{c}\right) \rightarrow Q_{c}\left(T_{h}\right)\right]_{\text {rev }}=-\frac{Q_{c}}{T_{c}}+\frac{Q_{c}}{T_{h}}
\end{gathered}
$$

Implicit in Equations (15) and (17) is the fact that when the exchange of heat takes place between two bodies of essentially the same temperature, the entropy change is zero, i.e.

$$
\Delta S[Q(T) \rightarrow Q(T)]=-\frac{Q}{T}+\frac{Q}{T}=0
$$

\subsection{Two more Transformations}

To complete the set of values that defines Clausius' second law thermodynamics we need to add to those given by Equations (14) to (17) the one corresponding to the irreversible transformation of heat from high to low temperature: $\left[Q_{c}\left(T_{h}\right) \rightarrow Q_{c}\left(T_{c}\right)\right]_{i r}$ as well as the one associated to the irreversible transformation of work into heat: $[W \rightarrow Q(T)]_{i r r}$.

These values were specified by Clausius as follows: "If...a quantity of heat $Q$ is generated by any process such as friction, and this is finally imparted to a body of temperature $T$, the uncompensated transformation thus produced has the value $Q / T$. Again, if a quantity of heat $Q$ has passed by conduction from a body of temperature $T_{1}$ to another of temperature $T_{2}$, then the uncompensated transformation is $Q\left(\left(1 / T_{2}\right)-\left(1 / T_{1}\right)\right)$ " (Clausius, 1879, p. 214).

In our terminology these values are represented as follows:

$$
\begin{gathered}
\Delta S\left[W \rightarrow Q\left(T_{h}\right)\right]_{i r r}=Q / T_{h} \\
\Delta S\left[Q_{c}\left(T_{h}\right) \rightarrow Q_{c}\left(T_{c}\right)\right]_{i r r}=Q_{c}\left(\frac{1}{T_{c}}-\frac{1}{T_{h}}\right)
\end{gathered}
$$

Table 1. Clausius' second law thermodynamics finds definition in the values or entropy changes it assigns to the different transformations from heat to work, work to heat, and from heat of one temperature to another, as well as by the notions subsumed by these values

\begin{tabular}{ll}
\hline Transformation & Clausius Formulation \\
\hline$\left[Q\left(T_{h}\right) \rightarrow W\right]_{r e v}$ & $-\frac{Q}{T_{h}}$ \\
{$\left[W \rightarrow Q\left(T_{h}\right)\right]_{r e v}$} & $\frac{Q}{T_{h}}$ \\
{$\left[W \rightarrow Q\left(T_{c}\right)\right]_{i r r}$} & $\frac{Q}{T_{c}}$ \\
{$\left[Q_{c}\left(T_{h}\right) \rightarrow Q_{c}\left(T_{c}\right)\right]_{r e v}$} & $-\frac{Q_{c}}{T_{h}}+\frac{Q_{c}}{T_{c}}$ \\
{$\left[Q_{c}\left(T_{c}\right) \rightarrow Q_{c}\left(T_{h}\right)\right]_{r e v}$} & $\frac{Q_{c}}{T_{h}}-\frac{Q_{c}}{T_{c}}$ \\
{$\left[Q_{c}\left(T_{h}\right) \rightarrow Q_{c}\left(T_{c}\right)\right]_{i r r}$} & $-\frac{Q_{c}}{T_{h}}+\frac{Q_{c}}{T_{c}}$ \\
\hline
\end{tabular}


The entropy changes given by Equations (14)-(17) and (19) and (20) appear in Table 1. They define Clausius formulation of the second law of thermodynamics.

\subsection{A Closer Look at Clausius' Transformations}

Additional knowledge to that advanced in Section 2.4 regarding the processes behind Clausius' transformations will be here provided. Of particular interest on reason of the role it plays in future arguments is the usually overlooked complex nature of the transformation of heat into work.

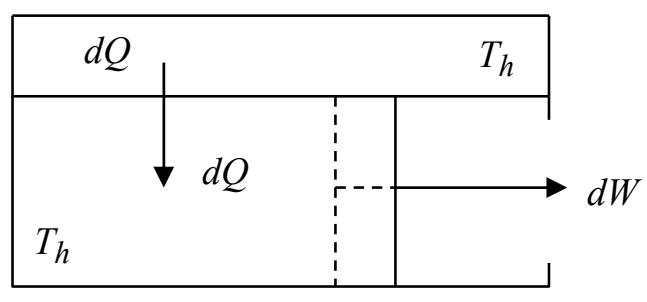

Figure 3. The isothermal and reversible expansion depicted transforms an amount of heat $d Q$ into an equivalent amount of work $d W$

The following argument makes reference to process $A E$ of Figure 2(b), identified in section 2.4 as the one responsible for the work output of the cycle via transformation $\left[Q\left(T_{h}\right) \rightarrow W\right]_{r e v}$. This process has been depicted in Figure 3. In it the ideal gas acting as variable body transforms quantitatively into work $(d W)$ an amount of heat $(d Q)$ absorbed from the hot reservoir. The very first thing that has to be understood in regard to this process is that before any amount of work $d W$ can be delivered to the mechanical reservoir, the equivalent amount of heat $d Q$ has to flow from the heat reservoir to the gas.

This argument leads to the realization that there are actually two transformations taking place in the said process: the reversible transfer of heat from the hot reservoir to the gas, and the transformation of this heat into work by the reversible expansion of the gas. In reference to a finite amount of heat $Q$ and its equivalent amount of work $W$, the situation just described can be represented as follows

$$
\begin{aligned}
& \Delta S_{\text {Clausius }}[\text { process } A E]_{\text {rev }}=\Delta S\left[Q\left(T_{h}\right) \rightarrow Q\left(T_{h}\right)\right]_{\text {rev }}+\Delta S\left[Q\left(T_{h}\right) \rightarrow W\right]_{\text {rev }} \\
& =0+\Delta S\left[Q\left(T_{h}\right) \rightarrow W\right]_{\text {rev }}
\end{aligned}
$$

The zero entropy change substituted in Equation (21) comes from the fact indicated by Equation (18) that any heat transference between bodies of essentially the same temperature takes place with a zero entropy change. Being this so, the entropy change for process $A E$ is solely and uniquely determined by the entropy change of the heat-towork transformation there taking place. To evaluate it all we have to do is to replace $\Delta S\left[Q\left(T_{h}\right) \rightarrow W\right]_{r e v}$ with its actual value. No problem here. All we have to do is to look back at Table 1 . There we find that the value in question amounts to $-Q / T_{h}$. Therefore

$$
\Delta S[\text { process } A E]=-Q / T_{h}
$$

The previous argument demonstrates that the entropy change given by Equation (22) is a total entropy change, i.e. it corresponds to the universe of process $A E$ and as such it includes the $-Q / T_{h}$ entropy change sustained by the hot reservoir in releasing the amount of heat $Q$, the $Q / T_{h}$ entropy change of the ideal gas in absorbing it, and the $-Q / T_{h}$ entropy change of the actual transformation of this heat into work by the opposed volume increase of the gas. Equation (22) unequivocally expresses the fact that for Clausius' second law thermodynamics the universe of this process is actually negentropic, meaning that a net total entropy decrease proportional to the amount of work produced has taken place.

The previous discussion makes evident the fact that the entropy change for the actual transformation of heat into work is a term separate and distinct from that associated to the release of this heat by the hot reservoir.

The total entropy change for isothermal and reversible process $E A$, the inverse of the one just considered, can now be written as follows 


$$
\begin{aligned}
& \Delta S_{\text {Clausius }}[\text { process } E A]_{\text {rev }}=\Delta S\left[Q\left(T_{h}\right) \rightarrow Q\left(T_{h}\right)\right]_{r e v}+\Delta S\left[W \rightarrow Q\left(T_{h}\right)\right]_{\text {rev }} \\
& =0+\Delta S\left[W \rightarrow Q\left(T_{h}\right)\right]_{\text {rev }}
\end{aligned}
$$

Substitution in Equation (23) of the $Q / T_{h}$ value given by Table 1 for the reversible transformation of work into heat leads us to:

$$
\Delta S_{\text {Clausius }}[\operatorname{Pr} \text { ocess EA }]_{\text {rev }}=Q / T_{h}
$$

The argument just given demonstrates that the $Q / T_{h}$ entropy change assigned by Equation (24) to process $E A$ is a total entropy change on reason of it combining the $Q / T_{h}$ entropy change associated to the actual transformation of work into heat produced along the compression, with the $-Q / T_{h}$ and $Q / T_{h}$ entropy changes sustained by the gas and the heat reservoir in respectively releasing and absorbing the amount of heat $Q$. This indicates that in Clausius' thermodynamics the universe of process $E A$ is entropic as in it entropy is produced in an amount proportional to the work dissipated.

Let us now center our attention on the concatenation of processes $E B-B C-C D-D A$ as represented in Figure 2(b). As should be recalled, this concatenation is the one responsible for bringing forward the reversible transfer of $Q_{c}$ from the hot to the cold reservoir. Its entropy change in terms of its constitutive processes can be written as follows:

$$
\begin{aligned}
& \Delta S_{\text {Clausius }}[E B-B C-C D-D A]_{\text {rev }}=\Delta S[E B]_{\text {rev }}+\Delta S[B C]_{\text {rev }}+\Delta S[C D]_{\text {rev }}+\Delta S[D A]_{\text {rev }}= \\
& \Delta S[E B]_{\text {rev }}+\Delta S[C D]_{\text {rev }}
\end{aligned}
$$

The isentropic nature of processes $B C$ and $D A$ explain their absence in the final right hand side term of the previous equation. The fact that processes $E B$ and $C D$ are of the same respective nature as processes $A E$ and $E A$ allows us, in line with Equations (21) and (23), to write the previous equation in the following manner

$$
\begin{aligned}
& \Delta S_{\text {Clausius }}[E B-B C-C D-D A]_{\text {rev }}=\Delta S\left[Q_{c}\left(T_{h}\right) \rightarrow Q_{c}\left(T_{h}\right)\right]_{\text {rev }}+\Delta S\left[Q_{c}\left(T_{h}\right) \rightarrow W_{c}\right]_{\text {rev }} \\
& +\Delta S\left[W_{c} \rightarrow Q_{c}\left(T_{c}\right)\right]_{\text {rev }}+\Delta S\left[Q_{c}\left(T_{c}\right) \rightarrow Q_{c}\left(T_{c}\right)\right]_{\text {rev }}
\end{aligned}
$$

The combination of the notion expressed by Equation (18) with the fact that in Clausius' thermodynamics “....the passage of the quantity of heat $Q_{c}$ from $T_{h}$ to $T_{c}$ has the same equivalence-value as a double transformation of the first kind, viz. the transformation of the quantity $Q_{c}$ from heat of temperature $T_{h}$ into work, and again out of work into heat of temperature $T_{c}$." (Clausius, 1879, p. 101) allows us to write

$$
\begin{aligned}
& \Delta S_{\text {Clausius }}[E B-B C-C D-D A]_{\text {rev }}=\Delta S\left[Q_{c}\left(T_{h}\right) \rightarrow Q_{c}\left(T_{c}\right)\right]_{\text {rev }}= \\
& \Delta S\left[Q_{c}\left(T_{h}\right) \rightarrow W_{c}\right]_{r e v}+\Delta S\left[W_{c} \rightarrow Q_{c}\left(T_{c}\right)\right]_{r e v}=\left(-Q_{c} / T_{h}\right)+\left(Q_{c} / T_{c}\right)
\end{aligned}
$$

Equation (26) allows us to see that in addition to the zero contributions of isentropic processes $B C$ and $D A$, the entropy change of the concatenation includes all the other entropy changes in it taking place. In process $E B$ we have the compensated entropy changes of the gas and hot reservoir in exchanging the amount of heat $Q_{c}$, plus that of magnitude $-Q_{c} / T_{h}$ associated to the actual transformation of heat into work. In process $C D$ we have the compensated entropy changes of the gas and cold reservoir in exchanging the amount of heat $Q_{c}$, plus that of magnitude $Q_{c} / T_{c}$ associated to the actual transformation of work into heat. This is what makes the entropy change assigned by Equation (27) to the said concatenation a total entropy change.

The fact that combined, processes $E B-B C-C D-D A$ and $A E$ define one cycle in the operation of the reversible heat engine depicted in Figure 2 allows us to realize that the total entropy change of the latter will be determined by the summation of the total entropy changes of the two former processes. Furthermore, on reason of the entropy changes of these two processes being quantified, as made evident by Equations (21) and (27), by the respective entropy changes of transformations $\left[Q\left(T_{h}\right) \rightarrow W\right]_{r e v}$ and $\left[Q_{c}\left(T_{h}\right) \rightarrow Q_{c}\left(T_{c}\right)\right]_{r e v}$, the cycle's total entropy change will be ultimately determined by the summation of the entropy changes of these two transformations, as follows

$$
\Delta S_{\text {Clausius }}[\text { Cycle }]_{\text {rev }}=\Delta S\left[Q\left(T_{h}\right) \rightarrow W\right]_{\text {rev }}+\Delta S\left[Q_{c}\left(T_{h}\right) \rightarrow Q_{c}\left(T_{c}\right)\right]_{\text {rev }}=-\frac{Q}{T_{h}}+\left[-\frac{Q_{c}}{T_{h}}+\frac{Q_{c}}{T_{c}}\right]=0
$$

Or, via a simple regrouping of its terms in accord to Equation (1), as follows 


$$
\Delta S_{\text {Clausius }}[\text { Cycle }]_{\text {rev }}=-\frac{Q_{h}}{T_{h}}+\frac{Q_{c}}{T_{c}}=0
$$

Equations (28) and (29) are mathematical expressions for the second law understood as "...in a reversible cyclical process the total value of all the transformations must be equal to nothing." (Clausius, 1879, p. 102)

\subsection{The Law of Increasing Entropy}

That the entropy changes of the two transformations in a reversible cycle combine to a value of zero allows referring to them as compensated transformations: the entropy increase associated to the transformation of heat from the hot to the cold reservoir being precisely compensated by the entropy decrease associated to the transformation of heat into work. Imagine now a portion of $Q_{h}$ reaching the cold reservoir having previously bypassed -say on reason of imperfect insulation- the circuit of changes of the variable body. In doing so, it also bypasses its opportunity to deliver the amount of work it had otherwise been extracted from it. This work is the 'lost work' originally identified by Carnot as the distinctive characteristic of what we now call an irreversible operation (Carnot, 1824/1977, p. 14). Under this condition the positive entropy change associated to this heat transfer finds no compensating negative entropy change. The fact that the entropy changes for the remaining portion of heat -the one getting to the cold reservoir through the variable body- and the work from it produced are compensated leaves the uncompensated entropy change as the sole result of the operation. It makes it an entropic operation. The mathematical expression of these considerations is the matter of the argument that follows.

If we let $Q_{i r r}$ represent the portion of $Q_{h}$ arriving in a direct fashion to the cold reservoir, then $Q^{\prime}=Q_{h}-Q_{i r}$ is the amount of heat entering the reversible cycle of changes of the variable body. From $Q^{\prime}$ an amount of work $Q$ is produced and the rest, $Q_{c}^{\prime}$ is released to the cold reservoir. The total entropy change for this irreversible operation can now be written as follows

$$
\Delta S_{\text {Clausius }}[\text { Cycle }]_{i r r}=\Delta S\left[Q_{i r r}\left(T_{h}\right) \rightarrow Q_{i r r}\left(T_{c}\right)\right]+\Delta S\left[Q\left(T_{h}\right) \rightarrow W\right]_{r e v}+\Delta S\left[Q_{c}^{\prime}\left(T_{h}\right) \rightarrow Q_{c}^{\prime}\left(T_{c}\right)\right]_{\text {rev }}
$$

The fact that the last two transformations of the right hand side of the previous equation are the ones produced by the reversible cycle, and as such are compensated transformations, allows us to substitute by zero their combined entropy changes. If so, the total entropy change for this operation -the irreversible cycle- reduces to

$$
\Delta S_{\text {Clausius }}[\text { Cycle }]_{i r r}=\Delta S\left[Q_{i r r}\left(T_{h}\right) \rightarrow Q_{i r r}\left(T_{c}\right)\right]
$$

Substitution in Equation (31) of the value given by Table 1 for the irreversible transformation of heat allows us to see that this irreversible operation takes place with a positive entropy change:

$$
\Delta S_{\text {Clausius }}[\text { Cycle }]_{i r r}=\Delta S\left[Q_{i r r}\left(T_{h}\right) \rightarrow Q_{i r r}\left(T_{c}\right)\right]=-\frac{Q_{i r r}}{T_{h}}+\frac{Q_{i r r}}{T_{c}}>0
$$

Results of this sort led Clausius to conclude that the combined value of all the transformations taking place in an irreversible cycle is larger than zero, or equivalently, that "uncompensated transformations must always be positive." (Clausius, 1879, p. 213)

As quoted in Section 2.1, for Clausius "The relation" between the transformations taking place in cyclical processes is "...that which is to be expressed by the second Main Principle."

This "relation" -the subject matter of the "second Main Principle"- takes form in the notions subsumed by Equations (29) and (32). Combined they embody the message of the second law as applied to cyclical processes: "The algebraic sum of all the transformations which occur in a cyclical process must always be positive or in the limit equal to zero." (Clausius, 1879, p. 213) After extending his analysis beyond the confines of cyclical processes, Clausius was ready to forward the following statement: "The theorem which...was enunciated in reference to circular processes only... has thus assumed a more general form, and may be enunciated thus:- The algebraic sum of all the transformations occurring in any alteration of condition whatever can only be positive, or as an extreme case, equal to nothing." (Clausius, 1862, p. 211)

The previous statement was eventually re-expressed as: "The entropy of the world tends to a maximum", a proposition commonly known as the law of increasing entropy.

\subsection{Universal Statements and Counterexamples}

In mathematics a universal statement is one supposed to be true for all members of a set. They are usually expressed in terms of words such as 'all', 'given any', 'every', 'each', and mathematically, with the symbol $\forall$, meaning 'for all'. The truth of a universal statement can be assessed either by proving that it is always true, or by showing 
it false for at least one member of the set. Such a case is a counterexample. In the next section the universal statements subsumed by the second law will be disproved -falsified- by counterexample.

\subsection{The Logical Holes in Clausius' Second Law Thermodynamics}

Clausius' second law statement given at the end of Section 2.10 can be re-expressed in terms of the following universal contentions: 1) that all reversible processes take place with a zero total entropy change; 2) that all irreversible processes take place with a positive total entropy change. Let us now provide counterexamples to these contentions

1) In order to prove false Clausius contention that all reversible processes take place at constant total entropy let us consider the ideal-gas isothermal and reversible expansion [IRE] through which $600 \mathrm{~J}$ of heat transferred from the heat reservoir to the gas, with both of these bodies at $300 \mathrm{~K}$, is transformed into $600 \mathrm{~J}$ of work. The entropy changes for the two transformations there taking place $[Q(T) \rightarrow Q(T)]_{r e v}$ and $[Q(T) \rightarrow W]_{\text {rev }}$ are, respectively, zero and $-Q / T$. According to this the total or universe entropy change for this process is the following:

$$
\Delta S_{\text {Clausius }}[I R E]_{\text {rev }}=-Q / T==-600 / 300=-2 \mathrm{~J} / \mathrm{K}
$$

For the inverse of the previous process, i.e. for the ideal-gas isothermal and reversible compression [IRC] through which the $600 \mathrm{~J}$ of work expended in carrying on this process end up as $600 \mathrm{~J}$ of heat in the heat reservoir, the entropy changes for the transformations involved $[Q(T) \rightarrow Q(T)]_{r e v}$ and $[W \rightarrow Q(T)]_{r e v}$, are zero and $Q / T$. If so, the total entropy change for this process is the following:

$$
\Delta S_{\text {Clausius }}[\text { IRC }]_{\text {rev }}=Q / T=2 \mathrm{~J} / \mathrm{K}
$$

The fact that these non-zero total entropy changes corresponds to processes which are reversible negates the contention demanding a zero total entropy change from all such processes.

2) In order to disprove the contention that all irreversible processes take place with a positive total entropy change let us consider the following argument developed in reference to Figure 4.

The figure depicts that situation in which only one half of the amount of heat $Q$ released by the hot reservoir of temperature $T_{h}$ is converted into an equivalent amount of work via the isothermal and reversible expansion of an ideal gas. The other half finds, on reason of imperfect insulation, a direct, irreversible path to a cold reservoir of temperature $T_{c}, T_{c}\left\langle T_{h}\right.$.

For the total or universe entropy change $\left(\Delta S_{u}\right)$ of this process we can write the following expression:

$$
\Delta S_{u}=\Delta S\left[\frac{Q}{2}\left(T_{h}\right) \rightarrow \frac{Q}{2}\left(T_{h}\right)\right]_{r e v}+\Delta S\left[\frac{Q}{2}\left(T_{h}\right) \rightarrow W\right]_{r e v}+\Delta S\left[\frac{Q}{2}\left(T_{h}\right) \rightarrow \frac{Q}{2}\left(T_{c}\right)\right]_{i r r}
$$

In Equation (35) the first two terms of the right hand side correspond to the work producing path; the last one, to the irreversible transfer of $Q / 2$ from the hot to the cold reservoir. Proper substitution in the previous equation of the corresponding values according to Table 1 leads to

$$
\Delta S_{u}=0-\frac{Q}{2 T_{h}}-\frac{Q}{2 T_{h}}+\frac{Q}{2 T_{c}}
$$

From it we can set up the following inequality in order to investigate the condition to be satisfied for the universe of this irreversible process to access negative total entropy changes

$$
-\frac{Q}{T_{h}}+\frac{Q}{2 T_{c}}<0
$$

Its solution leads us to $\left.T_{c}\right\rangle\left(T_{h} / 2\right)$. Since by definition $\left.T_{h}\right\rangle T_{c}$, we can then conclude that for any $T_{c}$ such that $\left.\left.T_{h}\right\rangle T_{c}\right\rangle\left(T_{h} / 2\right)$ it is true that $\Delta S_{u}\left\langle 0\right.$. For example, let us assume the following values: $Q=1200 \mathrm{~J}, T_{h}=600 \mathrm{~K}$, and $T_{c}=400 \mathrm{~K}$. The substitution of these values in Equation (37) followed by performance of the of the indicated operations leads to a total or universe entropy change of $-0.5 \mathrm{~J} \mathrm{~K}^{-1}$. The fact that this irreversible process takes place with a negative total entropy change proves false the contention that all such processes take place with positive total entropy changes. 


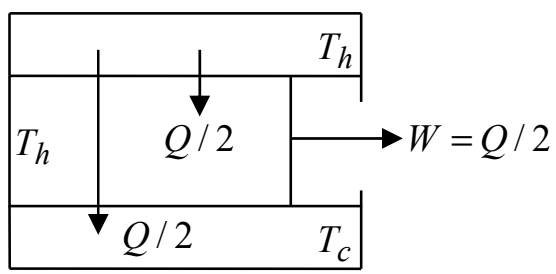

Figure 4. In this process only one half of the heat released by the hot reservoir ends up being transformed into work. The other half finds a direct, irreversible path to the cold reservoir. For any $T_{c} \in\left(T_{h} / 2, T_{h}\right)$ the total entropy change of this process turns out to be negative

The argument just given, it should be noted, also negates the certainty of the law of increasing entropy. For if Clausius formalism allows irreversible processes with positive as well as negative total entropy changes what proof do we have that the former kind predominates over the latter? With the same level of certainty anyone can claim that it is the latter kind the one predominating over the former, in which case the entropy of the universe will tend to a minimum; or to claim that at any given moment the contributions are equilibrated so the universe evolves along a constant total entropy path; or even claim that they alternate predominance so that the entropy path of the universe oscillates between positive and negative values. The fact that no proof to any of these effects seems to exist, disqualifies any assertion about the absolute validity of any of these scenarios (See Section 5.7, Part II). The simple example of Figure 4 has managed to replace Clausius' complete certainty about the entropy-condition of the universe by one of complete ignorance.

An additional inconsistency in Clausius' analysis is found embodied in the fact that the same total entropy change $\left(-Q_{c} / T_{h}\right)+\left(Q_{c} / T_{c}\right)>0$ is assigned in it to the reversible $\left[Q_{c}\left(T_{h}\right) \rightarrow Q_{c}\left(T_{c}\right)\right]_{r e v}$ and irreversible $\left[Q_{c}\left(T_{h}\right) \rightarrow Q_{c}\left(T_{c}\right)\right]_{i r}$ transfers of heat from a hot to a cold reservoir. According to this, transformation $\left[Q_{c}\left(T_{h}\right) \rightarrow Q_{c}\left(T_{c}\right)\right]$, as it takes place in reversible engine, has a dual nature: reversible on reason of the fact discussed in Section 2.4 that by coupling it with its inverse it is possible to restore the initial condition; irreversible on reason of its positive total entropy change (Section 2.9). This example, an ideal candidate for Aristotle's law of contradiction, also negates the role of the entropy change as the ultimate judge for the reversible and irreversible condition of a given process.

Let us finally point out that notable among the flaws is the fact that Clausius rule demanding a zero total entropy change for reversible processes was obtained by combining the non-zero total entropy changes (Section 2.9) of his reversible transformations.

In the second and final part of this work it will be seen how the second law of thermodynamics, as currently understood, emerges from an arbitrary correction of the flaws in Clausius' work.

\subsection{Conclusion}

The detailed discussion offered above has allowed us not only to identify the main logical flaws permeating Clausius' work, but also the construction of counter-examples to the two universal statements subsumed by his law of increasing entropy: that all reversible processes take place at constant total entropy, and that all irreversible processes take place with a positive total entropy change. In Clausius' second law thermodynamics neither all reversible processes take place at constant total entropy, nor all those irreversible occur with a positive total entropy change; If so, neither is the entropy change the ultimate judge for the reversibility, or lack of it, of thermodynamic processes.

\subsection{Final Comments}

This two part series supersedes all my previous work about the non-validity of the law of increasing entropy, among them (Íñiguez, 1999, 2011, and 2014).

Dedication: To my grandsons Nathan and Noah.

\section{References}

Callendar, H. L. (1911). The Caloric Theory of Heat and Carnot's Principle. Proc. Phys. Soc. (London), 23, 153189. http://dx.doi.org/10.1088/1478-7814/23/1/315

Carnot, S. (1824). Réflexions sur la puissance motrice du feu et sur les machines propres à développer atte puissance. Bachelier Libraire. 
Carnot, S. (1977). Reflections on the Motive Force of Fire (1824). In E. Clapeyron, R. Clausius, \& E. Mendoza (Eds.), Reflections on the Motive Force of Fire by Sadi Carnot and other Papers on the Second Law of Termodynamics. Peter Smith: Gloucester, MA, USA, 1977.

Clausius, R. (1862). On the Application of the Theorem of the Equivalence of Transformations to the Internal Work of a mass of Matter. Phil. Mag., S. 4, 24(159), 81-97, 201-213.

Clausius, R. (1879). The Mechanical Theory of Heat, Macmillan and Co., London, UK. Retrieved from https://archive.org/details/cu31924101120883

Íñiguez, J. (1999). A Revision of Clausius Work on the Second Law. Entropy, 1, 111-147.

Íñiguez, J. (2011). A Thermodynamic Impasse: A Constant Entropy Irreversible Process. Physical Chemistry: An Indian Journal, 6(3), 125-130.

Íñiguez, J. (2014). A Counterexample to the Second Law of Thermodynamics, Applied Physics Research, 6(3), 100-111. http://dx.doi.org/10.5539/apr.v6n3p100

Jaynes, E. T. (1988). In G. J. Erickson, \& C. R. Smith (Eds.), Maximum-Entropy and Bayesian Methods in Science and Engineering, 1. Kluwer Academic Publishers, Dordrecht, The Netherlands. Retrieved fromAvailable at: bayes.wustl.edu/etj/articles/ccarnot.pdf

Müller, I. (2007). A History of Thermodynamics: The Doctrine of Energy and Entropy. Springer, Berlin.

Planck, M. (1990). Treatise on Thermodynamics. New York, NY: Dover.

Purrington, R. D. (1997). Physics in the Nineteenth Century. New Brunswick, NJ: Rutgers University Press.

Truesdell, C. (1980). The Tragicomical History of Thermodynamics, 1822-1854. New York, NY: Springer-Verlag. http://dx.doi.org/10.1007/978-1-4613-9444-0

\section{Copyrights}

Copyright for this article is retained by the author(s), with first publication rights granted to the journal.

This is an open-access article distributed under the terms and conditions of the Creative Commons Attribution license (http://creativecommons.org/licenses/by/3.0/). 